\title{
Zum 100. Geburtstag von J.J. Burckhardt
}

Am 13. Juli 2003 durfte Johann Jakob Burckhardt, Titularprofessor für Mathematik an der Universität Zürich, in beneidenswerter geistiger Frische seinen hundertsten Geburtstag feiern.

Zu Ehren des Jubilars lud das Mathematische Institut auf den 31. Oktober 2003 zu einem Festkolloquium ein. Das Programm dieses Kolloquiums bestand aus einer Würdigung des Jubilars durch Erwin Neuenschwander (Zürich) und aus zwei Vorträgen, deren Gegenstände das mathematische Wirken von J.J. Burckhardt umreißen: Günther Frei (Zürich) sprach über die Geschichte der Arithmetik der Algebren und Ralph Strebel (Fribourg) über die Beiträge Burckhardts zur Mathematischen Kristallographie.

J.J. Burckhardt habilitierte sich 1933 an der Universität Zürich. Er wurde 1942 zum Titularprofessor befördert und wirkte von 1945 bis zu seiner Pensionierung als Oberassistent am Mathematischen Institut der Universität Zürich. Generationen von Mathematik- und Lehramtsstudierenden haben ihn als kompetenten und gütigen Lehrer erlebt. Sein Wirken ging aber weit über die Grenzen der Universität Zürich hinaus. Für seine vielfältigen Leistungen im Dienste der Wissenschaft ist er sowohl von der Schweizerischen Mathematischen Gesellschaft als auch von der Naturforschenden Gesellschaft in Zürich zum Ehrenmitglied ernannt worden.

Mit diesem Sonderheft möchte die Redaktion der Elemente der Mathematik dem Jubilar herzlich gratulieren und gleichzeitig die beiden Vorträge von G. Frei und R. Strebel einem größeren Kreis von Interessenten zugänglich machen. Beide Vorträge nehmen hauptsächlich Bezug auf die mathematischen Leistungen des jungen Burckhardt. Nicht zu vergessen sind aber auch seine zahlreichen späteren Beiträge zur Geschichte der Mathematik und der Naturwissenschaften. Aus diesem Grunde wurde auch eine Arbeit von Andreas Verdun (Bern) über L. Eulers Einführung und Anwendung von Bezugssystemen in Mechanik und Astronomie in dieses Sonderheft aufgenommen, welche einen Bezug zu Burckhardts Wirken in der Euler-Edition herstellt.

Markus Brodmann

Martin Huber

Jürg Kramer 